Relations industrielles

Industrial Relations

\title{
Ascah, Louis, Les secrets de la préparation financière à la retraite
}

\section{Louis Tremblay}

Volume 52, numéro 2, 1997

URI : https://id.erudit.org/iderudit/051180ar

DOI : https://doi.org/10.7202/051180ar

Aller au sommaire du numéro

Éditeur(s)

Département des relations industrielles de l'Université Laval

ISSN

0034-379X (imprimé)

1703-8138 (numérique)

Découvrir la revue

Citer ce compte rendu

Tremblay, L. (1997). Compte rendu de [Ascah, Louis, Les secrets de la préparation financière à la retraite]. Relations industrielles / Industrial Relations, 52(2), 466-468. https://doi.org/10.7202/051180ar

Tous droits réservés @ C Département des relations industrielles de l'Universite Laval, 1997
Ce document est protégé par la loi sur le droit d'auteur. L’utilisation des services d'Érudit (y compris la reproduction) est assujettie à sa politique d'utilisation que vous pouvez consulter en ligne.

https://apropos.erudit.org/fr/usagers/politique-dutilisation/ 
ne met en évidence que l'une des nombreuses dimensions importantes de la performance organisationnelle. Par exemple, Berggren ("Lean Production: The End of History ?", Work, Employment and Society, vol. 7, $\mathrm{n}^{\circ} 2,1993,163-188$ ) suggère que le régime de production a causé une résistance croissante des syndicats locaux et du désillusionnement chez les travailleurs de NUMMI (un cas important dans l'argumentation de Pfeffer) et ceux de CAMI alors que ces deux entreprises avaient adopté des pratiques de gestion des ressources humaines innovatrices. Une autre étude (R. Rehder, "Saturn, Uddevalla and the Japanese Lean Systems: Paradoxical Prototypes for the Twenty-first Century ", The Journal of Human Resource Management, vol. 5, $\mathrm{n}^{\circ} 1,1994,1-31$ ) soulève que Saturn et Uddevalla, deux entreprises avant-gardistes en matière de gestion des ressources humaines, ont fait face à des difficultés financières (Volvo a annoncé la fermeture de l'usine Uddevalla en 1993). Deuxièmement, une étude en profondeur sur l'évolution dans le temps et sur les effets des diverses stratégies et tactiques concurrentielles des cinq entreprises les plus performantes de 1972 à 1992 citées au début (ou des entreprises primées au Baldrige Award) permettrait probablement de mettre en perspective la thèse avancée par Pfeffer. Un autre élément méthodologique à envisager, quoique négligé dans les illustrations de l'auteur, pourrait consister à recueillir avec une plus grande attention le point de vue des travailleurs en ce qui a trait à la façon dont ils sont "gérés". Ceci peut fournir de l'information utile pour évaluer l'impact des pratiques mises en ceuvre sur les relations avec les employés, leur satisfaction, leur motivation et surtout de "relâcher le pouvoir des ressources humaines".

Somme toute, Jeffrey Pfeffer atteint son objectif. En plus de bien informer le public à qui il s'adresse de manière provocante et convaincante, de susciter des débats dans le monde universitaire, il redonne espoir aux praticiens en gestion des ressources humaines car, désormais, les managers peuvent difficilement nier qu'ils ont la responsabilité et la possibilité d'entreprendre la route qui mène au développement d'un avantage stratégique difficile à imiter et durable: le pouvoir et la force des ressources humaines. Pour effectuer un premier pas dans cette direction, Pfeffer propose que le management adopte un langage approprié. Ainsi, l'expression héritée du passé "gérer les ressources humaines " pourrait peut-être désormais s'énoncer, telle qu'utilisée une fois dans le volume, "gérer avec les gens".

\section{FRANÇOIS VILLENEUVE}

Université du Québec en Abitibi-Témiscamingue

\section{Les secrets de la préparation financière à la retraite}

par Louis ASCAH, Sherbrooke : Éditions du CRP, 1996, 189 p., ISBN 2-92085997-8.

Ce livre a pour objectif de rendre accessible la préparation de la retraite aux personnes intéressées. L'auteur atteint son objectif. Tout en étant rigoureux, il permet au lecteur de se faire une bonne idée sur les différents aspects nécessaires à une bonne planification de la retraite, c'est-à-dire les besoins à la retraite, les modes d'épargne public et privé, ainsi que les placements pour faire fructifier cette épargne. Par un heureux mélange de textes et de notes techniques, il trace un juste portrait de la planification à la retraite. Ce livre sera utile au lecteur qui désire être bien informé pour prendre des décisions éclairées.

Il fournit des outils utiles pour permettre au lecteur de mieux comprendre des données qui sont transmises par les conseils en placement, les institutions financières ou les spécialistes en 
avantages sociaux. Plusieurs tableaux sont intégrés au texte et des exemples en permettent une meilleure compréhension.

Le livre est divisé en huit chapitres : les besoins à la retraite, votre régime de retraite à l'emploi, les régimes publics, l'épargne personnelle, les REÉR, les FEÉR et les rentes viagères, les marchés financiers, la théorie moderne des placements, et conseil pour les placements dans votre REÉR.

Les besoins à la retraite sont le point de départ. L'auteur mentionne avec raison que le revenu à la retraite doit être suffisant à la première année de la retraite mais aussi à toutes les années. La protection contre l'érosion du niveau de vie par l'inflation est importante.

Le chapitre deux "votre régime de retraite à l'emploi " est mon préféré. L'auteur y vulgarise certains aspects encore mal compris par la majorité des participants à un régime de retraite d'entreprise, appelé aussi régime complémentaire de retraite. L'intégration ou la coordination de la rente du régime de retraite à l'emploi au Régime de rentes du Québec ou au Régime de pensions du Canada et la rente acquise en cas de départ avant la retraite (la règle de $50 \%$ de contribution minimale de l'employeur), des sujets particulièrement difficiles, ne manquent pas d'explications et d'exemples pour en faciliter la compréhension.

$\mathrm{Au}$ chapitre trois "les régimes publics ", l'auteur présente les programmes de pension de sécurité de vieillesse et de supplément de revenu garanti, le futur programme de prestations aux aînés (qui doit remplacer les deux programmes précédents en 2001) et le Régime de rentes du Québec. L'auteur a inclus dans ce chapitre un rappel des recommandations des différents rapports faites au fil des années pour améliorer les régimes publics. Sur le futur programme de prestations aux aînés, il souligne à juste titre que les contribuables feront face à des taux marginaux effectifs d'imposition élevés ce qui pourrait avoir des ef- fets sur l'incitation à l'épargne. Le taux marginal effectif de taxation est la somme du taux marginal d'imposition et du taux de récupération de la prestation aux aînés.

Au chapitre quatre "l'épargne personnelle ", l'auteur fournit au lecteur certaines notions fondamentales: la relation entre les années de travail et les années de retraite, le jeu de l'intérêt composé, la détermination de l'épargne requise pour se retirer à un âge donné avec une rente indexée. Pour bien faire comprendre cette notion plutôt difficile, il montre l'effet des différentes hypothèses en les faisant varier une à la fois: d'abord par une indexation partielle de la rente, ensuite avec un rendement de $1 \%$ plus élevé, et finalement avec des augmentations de salaires plus faibles que prévues.

Au chapitre cinq " les REÉR, les FEÉR et les rentes viagères ", l'auteur expose en détails la logique et l'historique des règles applicables aux contributions admissibles à un REÉR ou à un régime privé de retraite ainsi que certaines notions utiles: le report des contributions, la contribution au REÉR du conjoint, les cotisations excédentaires, les allocations de retraite, l'achat d'une rente viagère, la conversion du REÉR en FEÉR, le compte de rente immobilisé et le fonds de revenu viager.

La dernière partie du livre traite des notions de base en placement nécessaires au lecteur qui veut bien suivre ses affaires et profiter en investisseur éclairé et critique des conseils en placement qui lui sont offerts un peu partout.

Dans le chapitre six "les marchés financiers, c'est pas sorcier!", l'auteur nous présente la relation entre le taux d'intérêt et le prix des obligations, la relation entre la valeur actuelle, le taux d'intérêt et le prix des actions, et les différentes classes d'actif. Malgré de grands efforts de vulgarisation, cette matière reste aride, particulièrement l'exposé sur le prix de l'action.

Au chapitre sept "la théorie moderne des placements, ce n'est pas sorcier non 
plus ! ", il aborde des notions primordiales pour tout investisseur, qu'il dispose d'un petit comme d'un gros portefeuille, qu'il aime le risque ou qu'il l'ait en aversion. Il insiste sur la diversification adéquate entre les différentes classes d'actif et parmi chacune de celles-ci. Il apporte des nuances importantes sur l'analyse fondamentale et mentionne que des études ont démontré que la plupart des gestionnaires n'arrivent pas à battre les indices de marché. Cependant il admet que ce n'est pas chose facile.

Au chapitre huit "conseils pour les placements dans votre REÉR ", l'auteur reprend certains des éléments plus en détails. Il donne certains exemples de diversification de portefeuille. Pour l'auteur, les fonds mutuels offrent une diversification intéressante pour l'investisseur. Il recommande l'achat de fonds indiciels. Comme il est difficile de battre le mar- ché, il est important d'éviter de payer des frais de gestion élevés. Dans les fonds mutuels indiciels, le gestionnaire ne tente pas de battre le marché, mais il cherche plutôt par ces placements à choisir des actions représentatives du marché de l'indice, et les frais de gestion y sont moins élevés. Un conseil, qu'il donne, est " qu'il n'y a pas de Père Noël ", l'investisseur doit faire preuve de jugement par rapport aux possibilités d'investir qui lui sont offertes.

En annexe, l'auteur présente des feuilles de calcul pour que le lecteur puisse estimer l'épargne nécessaire pour atteindre ses objectifs de revenus à la retraite. Il y joint les instructions et les tables nécessaires pour compléter cet exercice.

LOUS TREMBLAY

Régie des rentes du Québec

Actes du XXXII ${ }^{\mathrm{e}}$ congrès de l'Association canadienne des relations industrielles Proceedings of the XXXII ${ }^{\text {nd }}$ Conference of the Canadian Industrial Relations Association

\section{La mondialisation de l'économie et le travailleur The Globalization of the Economy and the Worker} (Sélection de textes / Selected Papers)

Éditê par / Edited by

Anthony Giles, Anthony E. Smith. Gilles Trudeau

ACRI/CIRA, Département des relations industrielles

Université Laval, Québec, Qc, Canada, G1K 7P4 tél. (418) 656-2468 téléc./Fax (418) 656-3175

1 vol., 1995, 330 p., 35 \$, ISBN 0-9694230-7-1, ISSN 0-227-0013 